\title{
Desempeño de instituciones que atienden a niños "de" y "en" la calle en Venezuela
}

\author{
Rodríguez, Yudey* \\ * Magister en Ciencia Política (Universidad Sim ón Bolívar-Caracas). Lic. en Ciencia \\ Política (Universidad Fermín Toro/Barquisimeto-Lara). Investigadora asociada a la Unidad \\ de Políticas Públicas-USB. Valle de Sartenejas, Baruta - Miranda, Edif. Física y Electrónica \\ I, Piso I, Universidad Sim ón Bolívar. Telfs. (0212) 9063612. E-mail: yrodrig@usb.ve
}

\section{Resumen}

Con el objeto de contribuir a la creación de un marco de referencia en materia de seguimiento y evaluación de instituciones que atienden niños "de" y "en" la calle en Venezuela, la investigación se orienta a evaluar el desempeño institucional de la Casa Don Bosco de Valencia durante el período 1986-2000. Para ello se diseñó un instrumento de evaluación que responde a las características propias de los Programas Familia y Escuela Taller implementados en la Casa Don Bosco. Los resultados de la investigación indican que la institución ha ejercido un papel fundamental en la formación de cientos de niños y jóvenes "de" y "en" la calle, logrando mantenerse a través del tiempo a pesar de la continua reducción del presupuesto. Sin embargo, existen algunas limitaciones que deben ser superadas para garantizar un mayor desempeño institucional, las cuales están relacionadas con: falta de claridad en la definición de objetivos, alta tasa de deserción escolar comparada con la tasa de educación básica nacional, ausencia de informes anuales de gestión y de un plan nutricional elaborado por especialistas en la materia.

Palabras clave: Desempeño institucional, niños "de" y "en" la calle, evaluación de programas, programas sociales.

\section{Performance of Institutions Working With Both Street Children and Children in the Street, in Venezuela}

\begin{abstract}
This research was oriented towards evaluating the institutional performance of the "Don Bosco" House in Valencia from 1986 to 2000, with the objective of creating a frame of reference in the area of follow-up and evaluation of institutions that deal with street children in Venezuela. In order to do this, an evaluative instrument was designed that
\end{abstract}


responded to the characteristics of the Family and School-Workshop Program implemented in the "Don Bosco" House. The results indicated that this institution had performed a fundamental role in the formation of hundreds of street children and youth , maintaining itself over time even when there were funding restrictions. However, there are some limitations that should be overcome in order to guarantee greater institutional success, and which are related to: lack of clarity in the definition of objectives, a high level of school desertion compared with national statistics, the absence of yearly reports on the activity, and a nutritional plan elaborated by specialists in the area.

Key words: Institutional performance, street children, program evaluation, social programs.

Recibido: 02-03-12. Aceptado: 03-01-12

\section{I ntroducción}

Las familias venezolanas han experimentado un continuo deterioro del nivel de vida en las dos últimas décadas. Este deterioro ha sido mayor o menor dependiendo de los diferentes estratos sociales, pero en definitiva los más afectados han sido los estratos más pobres.

Uno de los sectores más afectados por la pobreza son los niños, pues han tenido que desarrollar diversas estrategias para sobrevivir. Muchos de ellos se han visto en la obligación y en la necesidad de salir a la calle a procurarse sus propios alimentos y en algunos casos los de su familia.

Durante las últimas tres décadas se han creado diversas instituciones que brindan atención a los niños que atraviesan serias dificultades, tanto en el sector público como en el privado. No obstante, a pesar de las acciones que se han tomado en materia de atención a los niños "de" y "en" la calle, el problema persiste. Podemos observar que sigue haciéndose sentir la presencia de niños deambulando por las calles de las principales ciudades del país.

Las cifras más recientes sobre el número de niños de la calle en Venezuela se encuentran en el Análisis de Situación de Menores en Circunstancias Especialmente Difíciles (MCED), realizado en 1992 por el Fondo de las Naciones Unidas para la Infancia (UNICEF) 1 . Se estimó que de una población de niños, niñas y adolescentes que llegaban a unos 7 millones, 4.800 .000 presentaban necesidades de atención preventiva; 9.583 se 
encontraban institucionalizados en centros del INAM; 1.584 .729 desarrollaban estrategias de sobrevivencia y 2.000 se ubicaban dentro de la categoría de niños de la calle ${ }^{2}$.

El signo de preocupación sobre esta situación no son las cifras estimadas para el año 1992, sino la tendencia a su crecimiento en la misma proporción que la pobreza, debido a que la mayoría de estos niños provienen de familias en situación de pobreza extrema, de barrios marginales y de zonas deprimidas económicamente.

La falta de un censo actualizado sobre la situación de los niños "de" y "en" la calle, se convierte en una de las principales limitaciones con la que debemos enfrentarnos quiénes investigamos sobre el tema. Dicha limitación representa un obstáculo no sólo para el investigador, sino también para el Estado, ya que se desconoce a ciencia cierta la población objeto de estudio y beneficiaria de las políticas y/o programas.

Durante los últimos años se han diseñado e implementado distintos programas orientados a brindar una mayor atención a estos niños y han surgido diferentes iniciativas por parte del sector público como del privado. Sin embargo, la revisión de la literatura demuestra serias limitaciones, en lo que se refiere a un proceso que es imprescindible para el mejoramiento de las instituciones que se dedican a atender a este tipo de niños, como lo es la evaluación.

De acuerdo a Dunn (1994: 405) "la evaluación desarrolla algunas funciones principales en el análisis de políticas: la primera y la más importante, es que la evaluación provee información confiable y válida sobre el desarrollo de las políticas, esto es, la magnitud en la cual las necesidades, valores y oportunidades han sido desarrolladas a través de la acción pública. Segundo, la evaluación contribuye a la clarificación y a la crítica de los valores que soportan la selección de metas y objetivos. Tercero, la evaluación puede contribuir a la aplicación de otros métodos de análisis de políticas".

Entre las principales razones que justifican la investigación se encuentran las siguientes: la necesidad de mejorar la situación que atraviesan los niños "de" y "en" la calle en nuestro país; el conocimiento limitado sobre el problema; el deterioro de las condiciones socio-económicas de la población; la ausencia de una política o Plan Nacional que agrupe y coordine las acciones orientadas a atender a estos niños, y finalmente, las reformas institucionales que se han dado en el sector público como la reestructuración del INAM y la entrada en vigencia de la Ley Orgánica para la Protección del Niño y el Adolescente, aprobada en 1998. 
Esta investigación se propuso como objetivo general evaluar el desempeño institucional de la Casa Don Bosco de Valencia, durante el período septiembre 1986 - julio 2000. Los objetivos específicos fueron: identificar los factores determinantes del problema de los niños "de" y "en" la calle en Venezuela, mediante el diseño y operacionalización de un modelo de políticas que responde a las características y particularidades del problema. Determinar si la Casa Don Bosco de Valencia estaba cumpliendo con los objetivos y servicios ofrecidos en el Proyecto Educativo Pastoral, mediante la evaluación de los Programas Familia y Escuela-Taller. Elaborar un marco de referencia y una metodología que sirviera de guía para la evaluación del desempeño de otras instituciones similares.

\section{Bases conceptuales $\underline{3}$}

Los principios conceptuales y metodológicos de esta investigación se fundamentan en el enfoque post-racionalista de las políticas públicas sistematizado por White (1994), haciendo énfasis en las siguientes premisas: el análisis de políticas públicas, como producto del debate político, el cual debe generar diferentes perspectivas o puntos de vista sobre los problemas y producir información relevante para la toma de decisiones en el proceso político. Por otra parte, debe estar dirigido al público y contribuir a la creación de espacios para la discusión de problemas relacionados con la gente. Finalmente, debe estudiar el rol de las instituciones como principal vínculo o enlace entre la gente y el gobierno. Dichas instituciones tienen la capacidad para generar cambios e implementar políticas mediante la producción de información útil sobre los intereses y valores de la gente.

Para la elaboración del modelo de políticas, se adoptaron algunas de las premisas conceptuales y metodológicas desarrolladas por MacRae (1985); relacionadas con la definición del problema expresado como un valor general final y la selección de variables contributivas que puedan ser manipuladas en el proceso político. Con el objeto diseñar políticas orientadas a la reducción o mejoramiento del problema planteado.

El modelo de políticas (Diagrama 1) se inicia con la definición del problema expresado como un valor general final y las variables contributivas expresadas como sistemas de políticas. El problema de los niños "de" y "en" la calle ha sido planteado como un fenómeno multivariado con diversos factores que lo condicionan. Estos factores son expresiones de los sistemas de políticas.

El modelo de políticas propone cuatro sistemas de políticas: económico, educativo, 
sociocultural e institucional. Estos sistemas han sido seleccionados respondiendo a la premisa de MacRae (1985) en lo relativo a la cercanía que debe existir entre las variables contributivas y el valor específico final. El objetivo es facilitar el marco de acción de las políticas orientadas a la solución o mejoramiento del problema. Cuando hablamos de variables contributivas o variables independientes, nos estamos refiriendo a los factores determinantes del problema, en este caso del problema de los niños de la calle.

\subsection{Definiciones básicas}

En este punto hemos incluido algunas definiciones que permiten establecer las principales diferencias que existen entre las diversas categorías de niños "de" y "en" la calle encontradas (niños de la calle 4 , niños en la calle, niñas de la calle y niños institucionalizados). La principal diferencia que existe entre estas definiciones es que representan diversos grupos con características muy particulares y están expuestos a diferentes tipos de riesgos o peligros. Los niños "de la calle" tienen como hábitat principal, permanente o transitorio la calle. Allí viven, duermen y desarrollan estrategias de

supervivencia. Los niños "en la calle" mantienen sus vínculos familiares, pernoctan regularmente en sus hogares y se ubican en la calle para trabajar. Las niñas "de la calle" representan una de las categorías que se encuentra expuesta a mayor riesgo por su condición de mujer y por los distintos problemas que se pueden generar a largo plazo, entre otros: embarazo precoz, abandono del hogar y prostitución. 


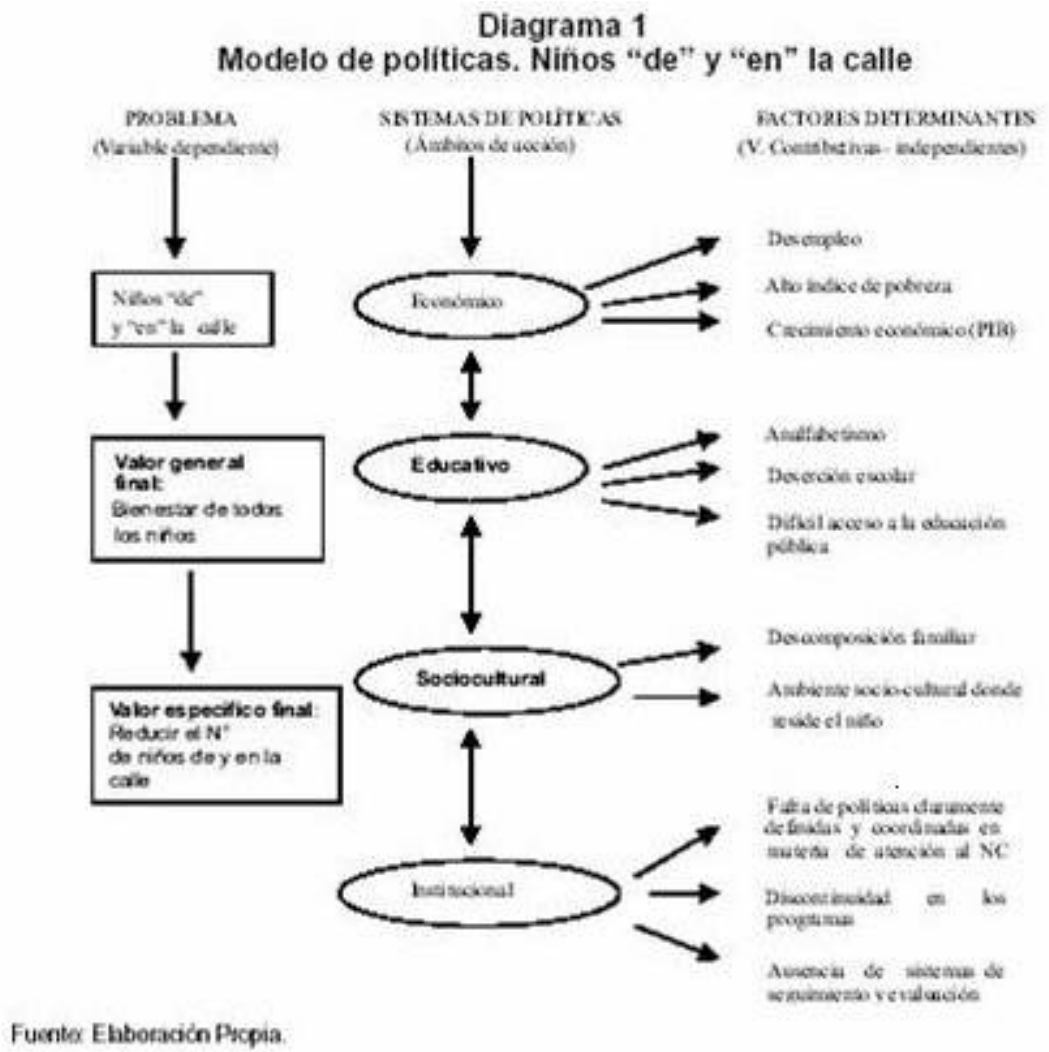

Cabe resaltar que, aunque las categorías señaladas representan grupos diferentes, la distancia entre una y otra es muy pequeña, existe la tendencia a cambiar rápidamente. Estos grupos están expuestos a una serie de condiciones que pueden llevarlos a convertirse definitivamente en ni ños de la calle. De igual forma, un niño institucionalizado, puede nuevamente convertirse en un niño "de" o "en" la calle. Esto dependerá del tipo de institución a la cual el niño ingrese, del tipo de atención que se le brinde y de las oportunidades que para él represente una nueva vida, marcada por normas y reglas a las que en la mayoría de los casos no está acostumbrado. De allí radica la importancia que el Estado debe atribuir al problema de los niños de la calle, en la calle e institucionalizados, haciendo énfasis en el fortalecimiento de las instituciones como principal medio de atención y fomentando políticas preventivas que garanticen efectivamente la recuperación de estos niños y su reinserción a la sociedad.

\section{Marco normativo institucional: atención a los niños “de" y “en" la calle}

Esta sección se dedica a la revisión del desempeño de instituciones que atienden a niños "de" y "en" la calle en Venezuela, bien sean de carácter público o privado, a través de las políticas y/o programas que se han desarrollado. El objeto de la sección es brindar una visión general sistematizada sobre el marco jurídico que las regula, el tipo de funciones que cumplen, los niveles de dependencia y el tipo de problemática que atienden. Para ello 
se establecieron tres niveles de estudio (Diagrama 2) que responden a los lineamientos y políticas que regulan su funcionamiento y se agruparon por sistemas de políticas con la finalidad de identificar el ámbito de acción de cada institución. De esta manera se facilita el diseño e implementación de políticas y/o programas orientados a brindar soluciones a los problemas que enfrenta la niñez en nuestro país.

En el primer nivel, intervienen los denominados sistemas de políticas generales, como son: el marco económico-fiscal (políticas macroeconómicas, políticas fiscales), el sistema político y el marco jurídico-legal. Estos sistemas tienen la particularidad de condicionar el funcionamiento de los sistemas de políticas específicos; en consecuencia, condicionan el desempeño de las instituciones involucradas con el problema de los niños "de" y "en" la calle.

El marco económico-fiscal, está representado por las diferentes políticas emanadas del gobierno que regulan el funcionamiento de todas las instituciones, tanto del sector público como del sector privado. El sistema político lo representa nuestro sistema de gobierno. El marco jurídico-legal está constituido por todas las leyes, decretos, ordenanzas y convenios que se han aprobado en el orden nacional e internacional en favor de los niños.

En el segundo nivel, intervienen los sistemas de políticas específicos. En este nivel, el Estado es el máximo organismo ejecutor de políticas orientadas a garantizar el bienestar de la sociedad. El Estado, como ente regulador, tiene entre sus principales funciones establecer los lineamientos generales que articulan el funcionamiento de las instituciones del sector público y privado. El sector público, lo representan las instituciones $u$ organismos del Estado. Siguiendo el orden de la estructura estatal, las instituciones encargadas de brindar atención a los niños "de" y "en" la calle son las siguientes: Ministerio de Educación, Cultura y Deportes; Ministerio de Salud y Desarrollo Social; (MSDS); Ministerio de Finanzas; Ministerio de Planificación y Desarrollo; Gobernaciones; Alcaldías; Instituto Nacional de Atención al Menor (INAM); Instituto Nacional de Nutrición (INN); Fundación del Niño y Fundación José Félix Ribas.

En el tercer nivel, se encuentran diversas instituciones que se han creado con la finalidad de brindar una respuesta a la problemática que confrontan los ni ños "de" y "en" la calle en Venezuela. Está representado por diversas organizaciones religiosas, organizaciones no gubernamentales (ONG's), fundaciones y asociaciones que atienden directamente a los niños. En este último nivel es donde se ubica la Casa Don Bosco de Valencia, institución cuya principal función es brindar atención a este tipo de ni ños. 


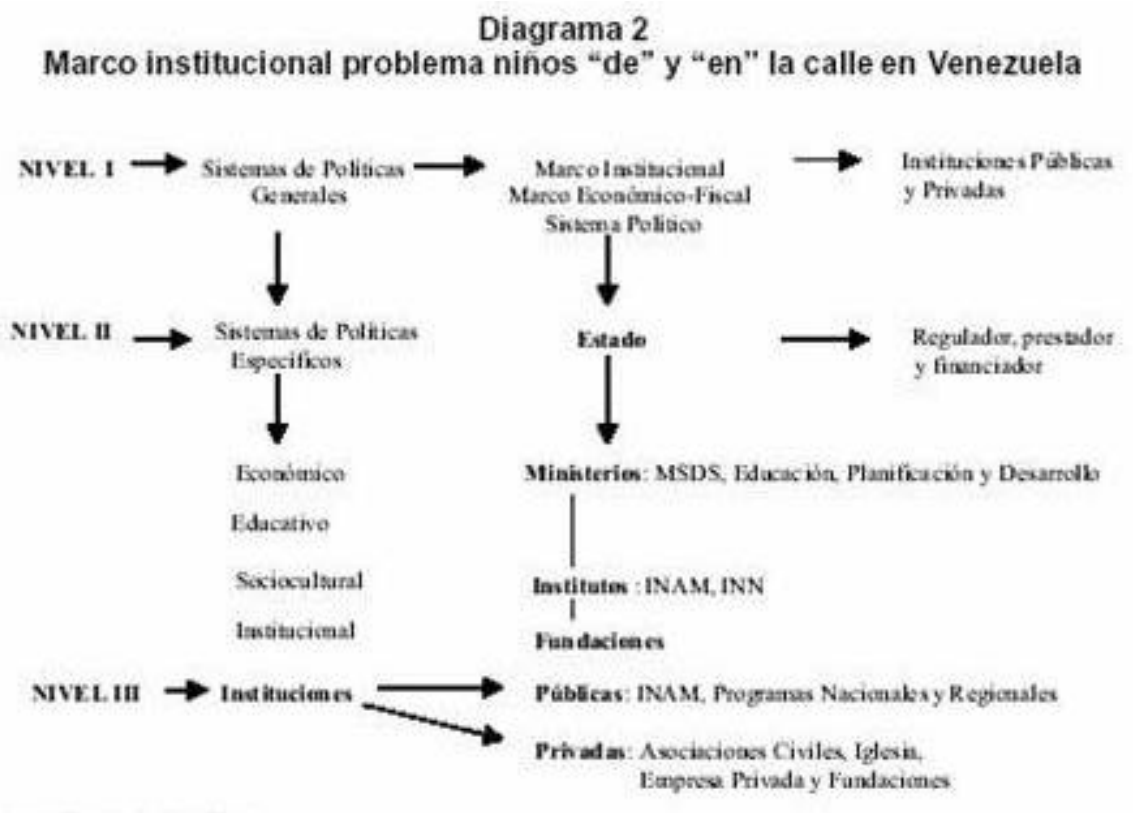

Fuente: Elboracín Propia

\subsection{Atención del Estado}

Para la revisión del tipo de atención que brinda el Estado venezolano a los niños "de" y "en" la calle, debemos considerar el rol que ha ejercido durante las últimas siete décadas frente al problema, tanto en el contexto internacional como en el contexto nacional.

En el contexto internacional el Estado venezolano ha dado varios pasos significativos en favor de la infancia y ha asumido los siguientes compromisos: Declaración de los Derechos del Niño (1969), Convención Internacional de los Derechos del Niño (1989) y la Cumbre Mundial en Favor de la Infancia (1990).

En el contexto nacional el Estado venezolano ha promulgado las siguientes figuras jurídicas: La primera Tabla de los Derechos del Niño (1936); Ley Tutelar del Menor (1980); Ley Aprobatoria de la Convención del Niño (1990); Conferencia Nacional sobre los Derechos del Niño (1991); Plan de Acción Intersectorial de Atención Integral a la Infancia y la Adolescencia (1994); Plan Maestro de Operaciones de Cooperación entre UNICEF y Venezuela (1996-2000); Ley Orgánica para la Protección del Niño y el Adolescente (2000); Ley sobre la Violencia contra la Mujer y la Familia (1998); Reglamento de Expedición de Partidas de Nacimiento (1998) y la Constitución de la República Bolivariana de Venezuela (1999), artículos 75, 78, y 79.

No obstante, a pesar de que se han dado pasos importantes en materia de atención a la 
niñez en Venezuela, todavía persisten algunos problemas relacionados específicamente con la efectividad de los programas que se han ejecutado, efectividad en términos de cobertura y calidad de servicios, ya que no responden a la magnitud del problema ni a su solución y/o reducción; la ausencia de sistemas de seguimiento y evaluación de dichos programas; la presencia de niños que viven en las calles de las principales ciudades del territorio nacional y la ausencia de un Plan Nacional que coordine la labor que realizan las instituciones que atienden la niñez en nuestro país.

En lo que se refiere a los programas del Estado en materia de atención a la niñez, hasta 1999 el INAM a través de sus distintas dependencias ha implementado tres (3) programas orientados hacia la atención del menor, a saber: Programa de Atención Preventiva, Programa de Atención al Menor en Situación de Abandono o Peligro, y el Programa de Atención al Menor con Necesidad de Tratamiento. Adicionalmente a estos programas también figura el denominado Programa Casa del Niño Trabajador (PCNT), aprobado en 1988, el cual ha sido implementado por diversos organismos del Estado a nivel nacional.

Entre las instituciones gubernamentales que se dedican a la atención de niños "de" y "en" la calle, así como también, de niños con otras problemáticas encontramos las que están adscritas al INAM y otras que son patrocinadas directamente por Alcaldías y Gobernaciones. Entre ellas podemos mencionar las siguientes: Carolina Uslar I; Casa del Menor Trabajador-Guárico; Casa del Niño Trabajador-Sucre y Trujillo; Casa del Niño Trabajador Panchito Mandefua (Alcaldía de Caracas); Casa Hogar INAM-Portuguesa; Casa Taller INAM: Falcón y Sucre; Centro de Atención Comunitaria INAM: Falcón, Mérida, Portuguesa y Puerto Cabello-Carabobo; Centro de Atención Dr. Luis Espelosín INAMDistrito Federal; Fundación del Niño y la Fundación José Félix Ribas.

\subsection{Atención de instituciones no gubernamentales}

Paralelamente a la respuesta dada por el Estado al problema de los niños "de" y "en " la calle en Venezuela, han surgido un gran número de instituciones con la iniciativa de diferentes sectores de la sociedad. Entre éstas podemos mencionar:

- Instituciones religiosas: Asociación Civil Fe y Alegría; Asociación Civil Red de Casas Don Bosco; Bemposta Nación de Muchachos y las Casas Hogares de las Hermanas del Buen Pastor.

- Organizaciones No Gubernamentales (ONG's): Asociación Muchachos de la Calle, Asociación Ayuda a un niño y los Hogares CREA.

- Instituciones dedicadas a la investigación y formación de personal capacitado para 
atender a los niños de la calle: Centros Comunitarios de aprendizaje (CECODAP); Centro Regional de Formación de Educadores de la Calle (CERFOCAL); Fundación Instituto de Capacitación e Investigación para el Recurso Humano que atiende al Niño y al Adolescente (FundaiCi) y el Instituto Universitario Pedagógico de Caracas.

- Instituciones que brindan apoyo financiero: Dividendo Voluntario para la Comunidad, Fundación Banco de Venezuela, Fundación Compañía Anónima de Teléfonos de Venezuela (CANTV) y Fundación TOGETHER.

- Instituciones internacionales que brindan apoyo financiero y asesoría como el Fondo de las Naciones Unidas para la Infancia (UNICEF), Organización Educativa, Científica y Cultural de las Naciones Unidas (UNESCO) y el Programa de las Naciones Unidas para el Desarrollo (PNUD).

\subsection{Ventajas y desventajas del actual marco institucional}

La aprobación de la Ley Orgánica de Protección del niño y del adolescente (LOPNA), ha dado un nuevo basamento legal a las instituciones que atienden el problema de los niños "de" y "en" la calle; presentamos algunas de las ventajas y desventajas que presenta el actual marco jurídico de atención a la niñez en Venezuela:

- Ventajas:

El actual marco institucional cuenta con un basamento jurídico favorable para la transformación y el mejoramiento de las instituciones que se dedican a brindar atención de los niños "de" y "en" la calle, bien sean del sector público o del sector privado, este es, la Ley Orgánica para la Protección del Niño y del Adolescente (LOPNA). Dicha ley representa todo un cambio de paradigma en cuanto a la concepción del niño como sujeto y objeto de derechos, respondiendo de esta manera a los lineamientos establecidos en la Convención Internacional de los Derechos del Niño (CIDN).

Una ventaja comparativa entre la LOPNA y la Ley Tutelar del Menor, es que la LOPNA permite la separación de los niños de acuerdo a sus problemáticas. Específicamente, en el caso de los niños infractores y los niños abandonados, prevé la instrumentación de dos sistemas para el tratamiento de los menores: uno de protección y otro penal, los cuales permiten la clasificación y especialización de las instituciones según la problemática que atienden.

- Desventajas:

La entrada en vigencia de la LOPNA no garantiza plenamente la solución de los 
problemas que afectan a la niñez en Venezuela. Por el contrario, la LOPNA advierte sobre las posibles dificultades que pueden presentarse con el proceso de reestructuración del INAM; la necesidad de coordinar los nuevos programas previo análisis y evaluación de los programas que se han implementado y la adopción de políticas coordinadas en el ámbito nacional, incorporando a todos los sectores involucrados (gobierno, empresa privada, iglesia, asociaciones, fundaciones, ONG's y comunidad en general).

El hecho de que contemos con un número significativo de instituciones gubernamentales y no gubernamentales de atención a los niños "de" y "en" la calle en nuestro país, no significa que todo está resuelto. Se requiere la implementación de sistemas de coordinación, seguimiento, control y evaluación para asegurar un mejor desempeño institucional.

La situación que atraviesa el país en general, caracterizada por altos índices de inseguridad, desempleo y pobreza afectan o condicionan el desempeño de las instituciones. Prueba de ello lo representa la Casa Don Bosco de Valencia, la cual se ha visto en la obligación de mantenerse y brindar los mismos servicios con menores recursos económicos y una consecuente reducción del número de niños atendidos.

\section{Evaluación del desempeño institucional de la Casa Don Bosco de Valencia}

Esta sección presenta en primer lugar una caracterización de la institución objeto de evaluación "La Casa Don Bosco de Valencia", y, en segundo lugar explica la metodología utilizada para la evaluación de su desempeño institucional.

\subsection{Caracterización de la Casa Don Bosco de Valencia}

La Casa Don Bosco de Valencia es una institución religiosa que pertenece a la Asociación Civil Red de Casas Don Bosco, coordinada por la Congregación Salesiana de Venezuela. Fue fundada por primera vez, en la ciudad de Los Teques en 1979, y en 1984 fue trasladada a Valencia, estado Carabobo. Actualmente funciona en la sede de la antigua Escuela de Agronomía Salesiana en la Redoma de Guaparo-Valencia.

La Casa Don Bosco de Valencia tiene como principal basamento teórico para el desarrollo de su labor el "Proyecto Educativo Pastoral" (PEP). El cual tiene entre sus principales objetivos brindar atención integral a niños y jóvenes en circunstancias especialmente difíciles y desarrollar una amplia acción educativo-pastoral. Está dirigido a niños y muchachos con edades comprendidas entre los 8 y 14 años que viven en situación de 
abandono, es decir, tienen la calle como ambiente de vida, son rechazados por la familia y la sociedad, y viven en extrema pobreza. También está dirigido a jóvenes mayores de 14 años que buscan una orientación en su vida.

Para la consecución de sus objetivos la Casa Don Bosco ha implementado tres programas: Programa Familia, Programa Escuela-Taller y el Programa Capacitación "Juventud y Trabajo". Para efectos de la evaluación se tomaron en consideración los dos primeros de manera integrada, ya que atienden a los mismos niños y se complementan mutuamente, a saber:

\section{a) Programa Familia.}

El objetivo general del Programa Familia es lograr que los muchachos de la Casa Don Bosco, realicen junto con los Salesianos y Laicos, una experiencia de hogar que les permita comprender la necesidad de esta vivencia humana como condición para un sano crecimiento, hasta lograr el estilo de Don Bosco, el surgimiento de un hombre nuevo, comprometido en la construcción de una sociedad nueva (Casa Don Bosco, 1998: 29).

b) Programa Escuela-Taller.

Es la expresión académica profesional de la Casa Don Bosco. En consecuencia, se establece una continua interrelación entre lo que se pretende con el Programa EscuelaTaller y lo que se quiere con el Programa Familia (Casa Don Bosco, 1998: 19).

EI PEP de 1998 es una versión mejorada del PEP de 1986; sin embargo, en el PEP 98 no aparecen claramente expresados los objetivos de este programa. Debido a esto hemos retomado lo planteado en el PEP de 1986 en cuanto al objetivo general de este programa:

Lograr que los muchachos de la Casa Don Bosco durante su permanencia en la misma, adquieran los instrumentos intelectuales y manuales que les permitan enfrentarse de nuevo a la vida con capacidad para autofinanciarse, con lucidez para penetrar al mundo del trabajo, con valores cristianos, con actitud de entrega y servicio en su comunidad de origen (Casa Don Bosco, 1986: 20).

El Programa Escuela-Taller tiene los siguientes objetivos específicos: Brindar estudios de educación básica (nivelación a 9o grado) y facilitar el aprendizaje laboral mediante prácticas sistemáticas en los talleres de herrería, electricidad, vivero, cerámica, carpintería, música, mecánica automotriz, informática, dibujo técnico, mecanografía y 
soldadura.

\subsection{Acciones metodológicas para la evaluación del desempeño institucional de la Casa Don Bosco de Valencia}

A continuación se explican los diseños elaborados para la evaluación del desempeño institucional de la Casa Don Bosco de Valencia y los resultados obtenidos con la aplicación del instrumento de evaluación. Este último constituyó la principal herramienta para la evaluación, permitiendo recopilar información relevante para determinar si la Casa estaba cumpliendo con los objetivos establecidos en los Programas Familia y Escuela-Taller.

\subsubsection{Diseño teórico de un programa de atención al niño "de" y "en" la calle}

Para la evaluación de los Programas Familia y Escuela-Taller de la Casa Don Bosco de Valencia se elaboró un programa ideal (marco teórico) que sirvió de guía para comparar y analizar los objetivos planteados en los programas de la institución. Este marco teórico para la evaluación está basado en los planteamientos de (Weiss, 1998) en lo referente a “la elaboración de una teoría como guía para la evaluación”. El marco teórico permite al evaluador ubicarse en "el deber ser" y lo que "debería ser" el programa, y a su vez, confrontar un programa ideal de atención a niños "de" y "en" la calle con la realidad.

El programa teórico diseñado para efectos de la evaluación contempla tres áreas: la primera, define como prioridad cubrir las necesidades básicas de atención que presentan los niños "de" y "en" la calle. Entre ellas se destacan: garantizar un techo seguro donde vivir; garantizar una alimentación balanceada que le permita al niño un sano desarrollo biológico; vestido y calzado acorde con su edad, talla y peso, y finalmente, la posibilidad de tener una familia que incluya una figura paterna o una persona que le inspire confianza y seguridad.

La segunda, establece las principales estrategias que se deberían tomar en materia educativa, destacándose: facilitar el acceso a la educación; brindar la posibilidad de que el niño estudie y culmine la educación básica; garantizar un proceso de formación educativa eficiente, que le permita nivelar sus conocimientos y aumentarlos; implementar simultáneamente con la educación básica cursos de capacitación en tareas productivas, que le permitan su incorporación al mercado laboral.

La tercera, contempla algunas de las acciones que debería desarrollar la Casa Don Bosco de Valencia en conjunto con otras instituciones del sector público y privado, con la 
finalidad de coordinar esfuerzos hacia la disminución y/o solución del problema de los niños "de" y "en" la calle. Entre estas acciones, se encuentran:

- Establecer convenios entre el Centro de Atención "Casa Don Bosco de Valencia", Gobierno y empresa privada que faciliten la incorporación de los muchachos en el mercado laboral.

- Implementar sistemas de seguimiento y evaluación de sus programas con el objeto de mejorar su desempeño institucional.

La mayoría de los objetivos que establecen los programas de la Casa Don Bosco enfatizan la formación basada en principios y valores religiosos. A pesar de que el Programa Familia prevé varias etapas para su ejecución, los objetivos para cada una de ellas son repetitivos y difusos. En los documentos revisados (PEP, 1996 y 1998) no aparecen definidos claramente los objetivos del Programa Escuela-Taller, sólo hace mención a la interrelación que existe entre los Programas Familia y Escuela-Taller.

\subsubsection{Evaluación por desempeño}

En este punto se explican los diferentes momentos de la evaluación que guardan relación con las etapas para la elaboración y aplicación del instrumento de evaluación, su estructura, los requerimientos de la evaluación, el tipo de diseño utilizado y el período que comprendió el trabajo de campo.

\section{- Etapas para la elaboración y aplicación del instrumento de evaluación.}

- Etapa I: Revisión de los contenidos que establece el Proyecto Educativo Pastoral, específicamente en los Programas Familia y Escuela-Taller. Para ello se tomó como principal referencia los objetivos que establecen cada uno de los programas. Se diseñó un programa teórico (programa ideal) que sirvió de guía para la evaluación y para comparar los objetivos que propone el diseño teórico y los programas de la Casa Don Bosco de Valencia.

- Etapa II: Identificación de las áreas de atención de los programas, con el objeto de diseñar un instrumento que respondiera a la realidad de la Casa y a las necesidades de los niños que en ella residen. De esta manera se facilitó la definición y selección de los indicadores para la evaluación.

- Etapa III: Asistencia y participación en el curso “Desempeño Institucional del Sector Público (DISP)", dictado en la Universidad Simón Bolívar, durante el período septiembre-diciembre 2000, con el objeto de seleccionar los indicadores que permiti ésen evaluar el desempeño institucional de la Casa. 
Durante el curso se elaboró la primera versión del instrumento de evaluación.

- Etapa IV: Discusión y aprobación de la versión definitiva del instrumento de evaluación con el Director de la Casa Don Bosco de Valencia.

- Etapa V: Aplicación del instrumento de evaluación. Recolección y sistematización de la información. Para ello fueron necesarias varias visitas a la institución, durante las cuales se fueron recabando el mayor número de datos posibles y se fueron recopilando cada una de las categorías que aparecen en el instrumento.

\section{- Estructura del instrumento de evaluación:}

- Datos básicos. Presenta datos que permiten identificar el tipo de labor que cumple la institución. Los indicadores para esta categoría son los siguientes: número de niños que ingresan a la Casa Don Bosco al año, número de niños que egresan de la Casa al año, tipo de niños atendidos ("de" o "en" la calle), edad promedio de los niños que ingresan por primera vez a la Casa, nivel educativo de la madre y capacidad instalada.

- Servicios. En esta categoría se encuentran los servicios que ofrece la Casa en las diferentes áreas de atención (salud, educación, formación religiosa, alimentación y capacitación laboral). Para la selección de los indicadores en cada una de las áreas antes mencionadas, se hizo especial énfasis en la selección de aquellos que pudieran medirse y que permitieran determinar si la Casa ha tenido un desempeño institucional positivo en el tiempo.

- Insumos. En esta categoría se incluyeron los insumos que la institución requiere para su funcionamiento (recursos humanos, presupuesto casa, presupuesto escuela, equipos e instalaciones).

- Procesos. Está constituida por los diferentes procesos (planificación, coordinación, supervisión, seguimiento y evaluación) que la Casa implementa para llevar a cabo su gestión.

- Requerimientos de la evaluación. La realización de la evaluación requirió del establecimiento de un grupo conformado por personal encargado de la ejecución del PEP en la Casa Don Bosco de Valencia. Específicamente el grupo estuvo conformado por el Director, el Administrador y un Profesor. Entre las principales actividades desarrolladas por el grupo se encuentran: colaboración en la revisión y diseño definitivo del instrumento de evaluación, revisión de las fuentes de información, coordinacción del proceso de recolección y sistematización de la información y revisión de registros para el período de la evaluación.

- Tipo de diseño. El diseño aplicado es cuasi-experimental de serie cronológica, sin grupo control $\underline{5}$. Se asumió que la intervención comprendía las actividades 
desarrolladas por la Casa, esperando que dichas actividades afectarían el desempeño en las áreas de servicios. También se asumió que la intervención se realizaba desde el primer año de actividad de la Casa. La unidad de análisis está representada por los años que comprenden el período 1986-2000. Se desarrolló un diseño en el que se tomaron datos para cada uno de los años en que la información se encontró disponible (desde el inició de la Casa). Con la finalidad de evaluar el desempeño institucional en el tiempo, mediante la comparación y el análisis de indicadores para cada una de las áreas que comprenden los Programas Familia y Escuela-Taller. Otros aspectos de la evaluación son meramente descriptivos.

Período de evaluación. Representa el tiempo utilizado para el trabajo de campo, este comprendió un período de seis meses (enero-junio 2001) y abarca las etapas siguientes:

- Etapa I: (2 meses). Revisión del diseño de evaluación con los directores de la Casa. Discusión de los indicadores del diseño. Elaboración del diseño definitivo.

- Etapa II: (2 meses). Recolección de la información y datos para la evaluación. Revisión de registros. Evaluación de las instalaciones.

- Etapa III: (2 meses). Análisis de los resultados.

\section{Análisis de los resultados}

En el transcurso de la evaluación, específicamente en la etapa de recolección y sistematización de la información para el instrumento, se pudo detectar la enorme importancia que tienen los registros para la implementación del sistema de seguimiento y evaluación de la Casa Don Bosco Valencia. Estos constituyen la principal fuente de información que permite al evaluador medir los resultados obtenidos a través de los programas en el tiempo. A continuación, presentamos los hallazgos en cada una de las áreas del instrumento de evaluación donde estaban disponibles los datos para todo el período evaluado, ya que existen algunas áreas como salud, nutrición y capacitación laboral en las que no se encontraron los datos requeridos para todo el período.

- Niños que ingresan anualmente a la Casa Don Bosco de Valencia (ver Gráfico 1).

Los datos obtenidos corresponden al número total de niños que ingresan a la institución anualmente, incluyendo las categorías: nuevos ingresos, alumnos que repiten el año escolar y alumnos que aprueban el año escolar. Durante el período 1986-1991 aumenta el número de niños que ingresan a la Casa Don Bosco. A partir de 1993 se inicia un aumento en el número de niños que ingresan a la institución manteniéndose estable hasta el año 
2000, fecha en que sufre una reducción considerable del número de ingresos, pasando de 214 en 1999 a 142 en el año 2000. Esta reducción tan significativa se atribuye a dos razones fundamentalmente: la primera a la falta de recursos económicos, ya que para el año 1999 la institución no recibió la colaboración que anualmente recibe de la gobernación, y la segunda por problemas de hacinamiento en los dormitorios.

- Nivel educativo de la madre (ver Gráfico 2).

Este indicador ha permitido confirmar que el analfabetismo constituye uno de los factores determinantes asociados a la problemática que envuelve a los niños "de" y "en" la calle, debido a que la mayoría de las madres de los niños de la Casa Don Bosco de Valencia durante el período estudiado (1986-2000), se ubicaron en la categoría analfabeta, manteniendo una tasa promedio entre $80 \%$ y 35\%, superada a partir de 1996 por la categoría $1^{\circ}$ a $6^{\circ}$ grado que se mantuvo entre $55 \%$ y $60 \%$ hasta el año 2000 . Otro dato importante que confirma que la tasa de analfabetismo de las madres de la Casa Don Bosco de Valencia es muy alta, lo representa la tasa de analfabetismo femenino en Venezuela, la cual durante el período 1980-1993 no supera el 16.9\% y ha mostrado una tendencia a la reducción ubicándose en 1993 en $8.89 \%$.

\section{Gráfico 1}

Ingreso de ninos a la casa Don Bosco de Valencia

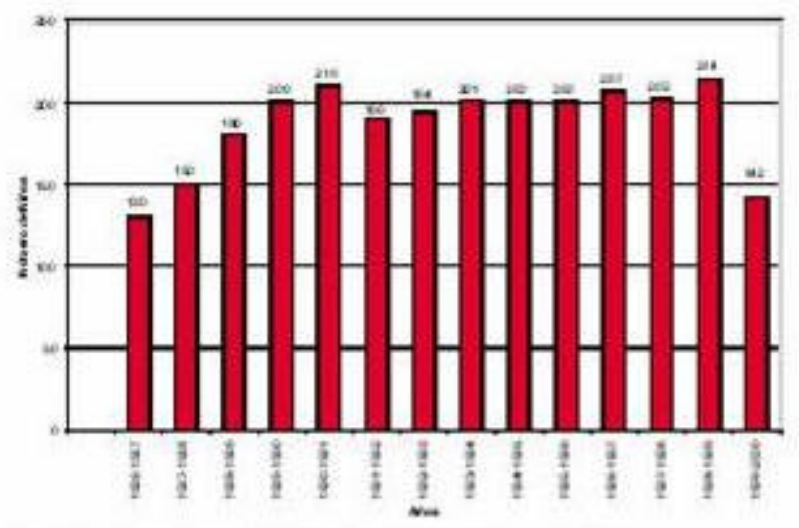

Fuente: Irstrumento de Evaluación Casa Don Bosco de Vałrncia 
Gräfico 2

Casa Don Bosco de Valencia

Nivel educativo de la madre

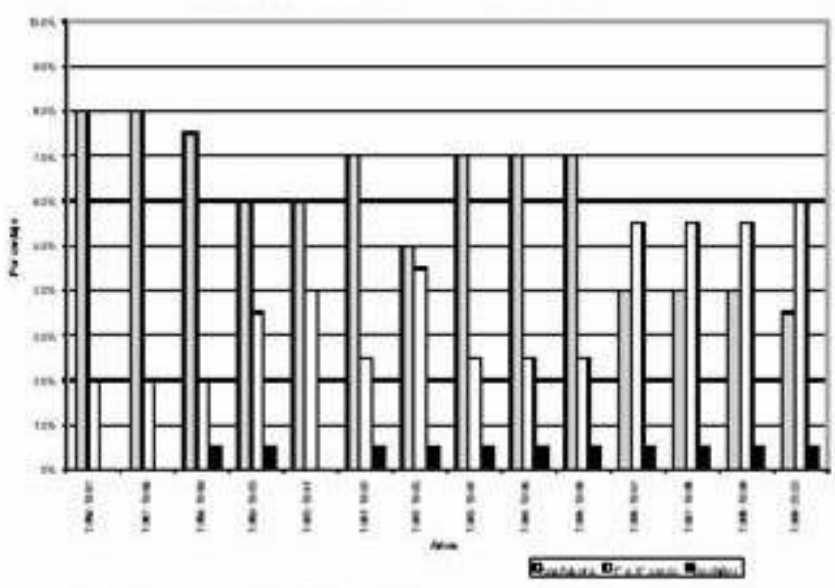

Fuente: Irstrumento de Evaluación Casa Don Bosoo de Vakenca

\section{Servicios}

- Salud.

No se tienen registros porque el servicio de enfermería se inició a partir del mes de septiembre del año 2000. Sin embargo, están empezando a organizar los registros, se han abierto historias para cada uno de los niños y se está brindando atención en las áreas de pediatría y dermatología una vez a la semana para los niños que así lo requieren.

- Alimentación.

En el área de pediatría para el momento de la evaluación se estaba elaborando un plan nutricional que contribuye al mejoramiento del servicio de atención alimenticia, ya que a pesar de que en la Casa Don Bosco de Valencia se ofrecen tres comidas diarias y una merienda, los menús no han sido elaborados bajo la supervisión y asesoramiento de un especialista en nutrición.

\section{- Educación.}

A pesar de que se encontraron los datos requeridos en el instrumento para los indicadores (tasa de deserción escolar y tasa de prosecución escolar) durante todo el período de estudio (1986-2000), la suma de estos porcentajes sobrepasa el 100\%. Esto puede atribuirse a dos razones fundamentalmente: la primera, que no fue incluida la tasa de repitencia, y la segunda, que existen fallas en el cálculo de los porcentajes suministrados por la institución. Esta última hipótesis, cobra mayor fuerza porque al sumar los porcentajes de deserción escolar (16.6\%) y prosecución escolar (98.0\%) para 
el año 1988-1989 el resultado da un porcentaje mayor al 100\%. Igualmente sucede al sumar los porcentajes de dichas tasas para los a ños: 1990-1994 y 1995-2000.

Otro hallazgo encontrado en materia educativa, lo representa el hecho de que la tasa de deserción escolar de la Casa Don Bosco de Valencia se mantuvo relativamente alta para la mayoría de los años en estudio, en comparación con la tasa de deserción de educación básica nacional (ver Gráfico 3). En tal sentido, la investigación sugiere estudiar a fondo las causas que han provocado tan altos índices de deserción escolar y diseñar estrategias dirigidas a su reducción.

- Capacitación laboral.

No se encontraron los datos requeridos para la evaluación. Sólo se tienen datos para el período 1997-2000, los cuales, revelan que para el año 1997-1998 de un total de 202 niños inscritos, 70 asistieron a los cursos que dicta la Casa Don Bosco de Valencia; en 1998-1999 de un total de 214 niños, sólo 71 asistieron a los cursos y en 1999-2000 de un total de 142 niños inscritos, sólo 72 asistieron a los cursos. De lo anterior, podemos inferir que un escaso número de niños asiste a los cursos que se dictan en la institución y que durante los últimos 3 años, se ha mantenido el mismo número de niños que asisten a los cursos, a pesar de la reducción del total de niños inscritos durante el último año escolar 1999-2000.

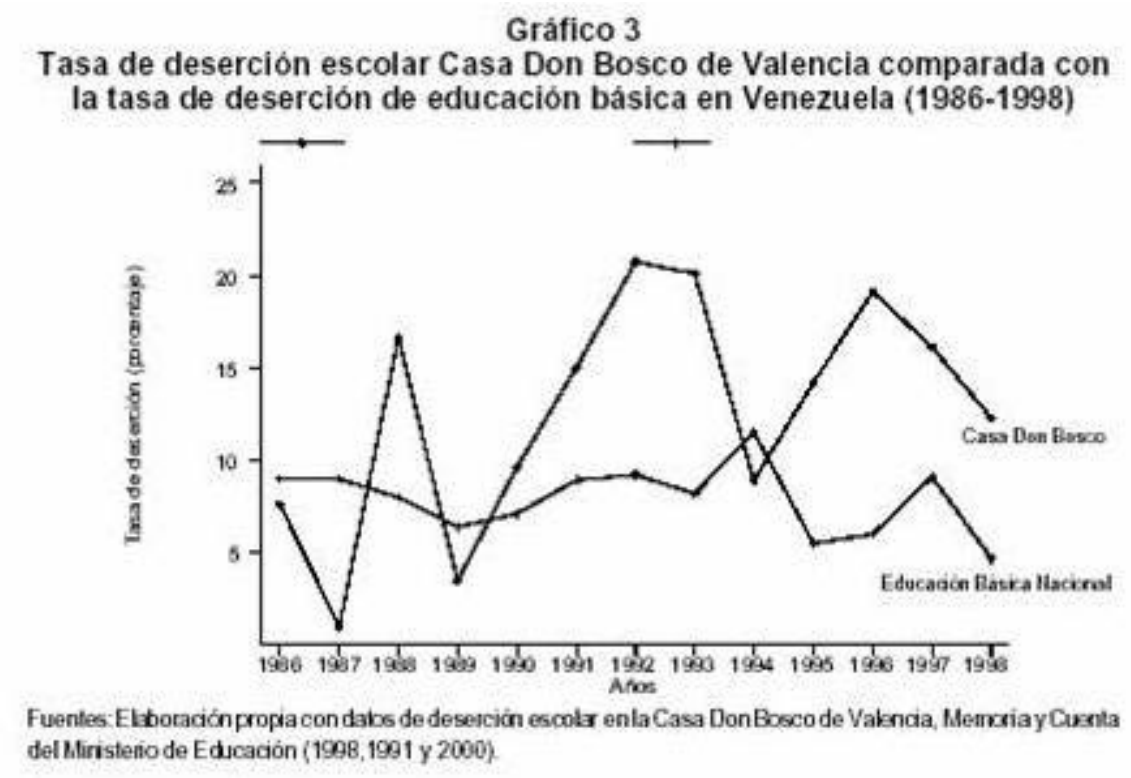

\section{Insumos}

- Recursos humanos. 
No se encontró la información solicitada para todo el período en estudio. Sólo se tienen datos para algunas categorías. Estas categorías corresponden al nivel académico de los profesores, número de profesores a tiempo completo y número de obreros que figuran en la nómina al año. Con la información encontrada no se pudo hacer mayor inferencia sobre la evolución de estos indicadores en el tiempo. En cuanto al nivel académico de los profesores durante los dos últimos años del estudio (1998-1999 y 1999-2000), se mantuvo el mismo número de técnicos y el número de profesionales aumentó de 15 a 19.

- Situación de equipos e instalaciones.

Para la evaluación de este indicador se establecieron tres categorías: bueno, regular y deficiente. En general, todas las instalaciones se ubican en la categoría bueno, a excepción de los baños que se ubican en la categoría deficiente y la enfermería en la categoría regular. Es por ello que consideramos necesario e imperativo iniciar acciones para mejorar las condiciones de los baños con el fin de evitar cualquier tipo de infecciones. En el área de enfermería se debe hacer un mayor esfuerzo por dotarla de los insumos necesarios para brindar una mejor atención a los niños.

\section{Procesos}

En lo relativo a los procesos de planificación, coordinación, supervisión, seguimiento y evaluación no se tiene un manual con las normas o estándares que regulan el funcionamiento de la Casa. Solamente se tienen normativas para la escuela. No se encontraron informes sobre el rendimiento anual de cada niño, lo cual impidió el análisis sobre la evolución del niño desde el momento en que ingresa a la Casa hasta que culmina su formación.

\section{Recursos financieros Casa Don Bosco de Valencia}

Para el análisis de los recursos financieros se tomaron los datos encontrados para todos los rubros presupuestarios de los Programas Familia y Escuela-Taller durante el período 1986-2000 y se llevaron las cifras presupuestarias a valores constantes a través del cálculo del deflactor del Producto Interno Bruto (PIB).

A diferencia del Programa Familia, el Programa Escuela-Taller presenta mayor orden y sistematización de los rubros presupuestarios y los datos se encontraron disponibles para toda la serie histórica. Estos datos revelan que el presupuesto de la escuela a precios constantes, sufre una reducción para la mayoría de los años estudiados, pasando de Bs. 
10.707.127 en 1986 a 1.966.050 en 1996. En consecuencia, se puede deducir que a medida que pasa el tiempo la escuela ha tenido menos ingresos para brindar los mismos servicios.

\section{Gasto per cápita Casa Don Bosco de Valencia}

El gasto per cápita ha sido calculado con los datos del presupuesto del Programa Escuela-Taller para el período 1986-2000. El presupuesto per cápita del Programa Escuela-Taller (ver Gráfico 4) permite verificar si los niños de la Casa Don Bosco perciben o se mantienen con la misma cantidad de dinero en el tiempo con relación a la inflación. En tal sentido, los datos relacionados con el presupuesto de ingresos y gastos del Programa Escuela-Taller, revelan que a medida que pasa el tiempo los gastos per cápita disminuyen.

Gráfico 4

Casa Don Bosco de Valencia

Programa Escuela-Taller

Gasto per cápita

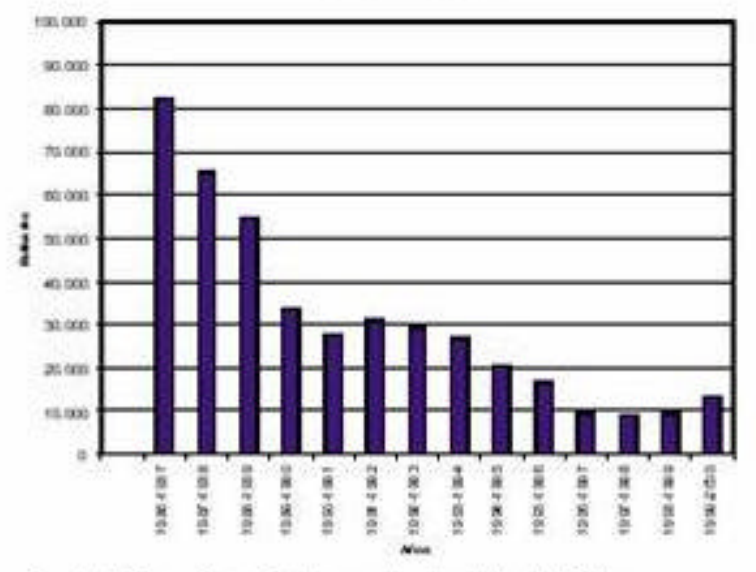

Fuanle: Elabcración pecpia de acuerdo a bs datos dotenidos.

\section{Conclusión}

El problema de los niños "de" y "en" la calle en nuestro país, está condicionado por diversos factores; entre ellos podemos mencionar: los altos índices de desempleo y pobreza; la ausencia de crecimiento económico sostenido; el analfabetismo; la deserción escolar; la ausencia de mecanismos que garanticen la escolaridad; la descomposición familiar; la falta de políticas claramente definidas en materia de atención al niño de la calle; la discontinuidad en la implementación de los programas y la ausencia de sus respectivos sistemas de seguimiento y evaluación.

El problema de los niños "de" y "en" la calle debe convertirse en un tema de orden 
prioritario en la agenda nacional, dada la importancia que reviste el futuro de nuestros niños. Es imperativo diseñar e implementar políticas públicas producto de la discusión y el consenso entre expertos y profesionales que representen los distintos sectores involucrados con la problemática, reconociendo desde el inicio que el problema de estos niños no puede combatirse con programas y/o políticas aisladas.

Conscientes de la importancia que tiene para el sector público y privado; así como también, para toda la comunidad en general la atención de los ni ños "de" y "en" la calle; debemos insistir en la necesidad de que se asignen recursos con la mayor efectividad y eficiencia posible, con la finalidad de lograr una mejor cobertura de los programas y más beneficios para todos los niños que confrontan serias dificultades en Venezuela.

La Casa Don Bosco de Valencia ha ejercido un papel fundamental en la formación de cientos de jóvenes o ni ños "de" y "en" la calle, logrando mantenerse a través del tiempo, a pesar de la continua reducción que han tenido en su presupuesto. Sin embargo, existen algunas limitaciones que deberían ser mejoradas para garantizar una mayor calidad de los servicios que presta. Entre ellas podemos mencionar las siguientes: en el área de los procesos, los objetivos que establecen los Programas Familia y Escuela-Taller no están claramente definidos, existen fallas en la sistematización y organización de los registros, no se tienen informes escritos sobre la gestión anual para cada uno de los años estudiados. En materia educativa la tasa de deserción escolar constituye un indicador significativo de que existen fallas en el sistema que deben corregirse, y en materia alimenticia la ausencia de un plan nutricional supervisado por un especialista, constituye otra limitación que se debe superar.

Dado que no existían estudios previos relacionados con la evaluación del desempeño institucional de la Casa Don Bosco de Valencia, esta investigación representa una primera aproximación en la que se lograron identificar los principales indicadores del desempeño institucional (tipo de niños que atiende la institución, número de niños atendidos en materia de salud, tipo de alimentación ofrecida, tasa de deserción escolar, tasa de participación en los talleres de capacitación laboral y formación religiosa). No obstante, no se establecieron estándares para cada uno de estos indicadores por dos razones fundamentalmente: en la primera, consideramos que todos los indicadores señalados son determinantes en el desempeño institucional de la Casa, y en la segunda se quería analizar cuál era el nivel inicial.

El instrumento de evaluación ha representado una herramienta fundamental para la evaluación del desempeño institucional de la Casa Don Bosco de Valencia. Asimismo, 
puede servir como marco de referencia para evaluar otras instituciones que se dedican a brindar atención a los niños que atraviesan serias dificultades, específicamente a ni ños "de" y "en" la calle en Venezuela.

\section{Notas}

1. El estudio fue hecho y publicado por UNICEF, con datos suministrados por el INAM.

2. No se puede precisar cuando comienza a existir un registro sobre datos relacionados con los ni ños "de" y "en" la calle, dado que es difícil encontrar estadísticas sobre este tema. Aquí se recurre a éstas, sin poder afirmar que sean las primeras. Tampoco hay mucha literatura publicada en Venezuela relacionada con el tema, sólo están las mencionadas en la bibliografía.

3. El desarrollo de este punto presentó algunos problemas dado la escasez de la literatura que pudiera soportarlo. Sólo se encontraron trabajos descriptivos sobre la situación que confrontan los niños "de" y "en" la calle, mas no trabajos relacionados con desempeño institucional, ni sobre evaluación de políticas de atención a estos niños.

4. Para consultar otras definiciones de ni ños de la calle, se recomienda revisar FUNDA-ICI/FUNDACION DE WAAL (1998).

5. Para revisar más detalles sobre este tipo de diseño, se recomienda consultar la obra de Campbell, D. y Stanley, J. (1966).

\section{Bibliografía Citada}

1. Asamblea Nacional. Ley Orgánica para la Protección del Niño y el Adolescente (LOPNA), Gaceta Oficial Extraordinaria 5.266 del 02/10/2000 de la República Bolivariana de Venezuela.

2. Campbell, D. y Stanley, J. (1966), Diseños experimentales y cuasiexperimentales en la investigación social. Amorrortu Editores, Buenos Aires. Argentina.

3. Casa Don Bosco (1998), Proyecto Educativo Pastoral, Valencia. Venezuela.

4. Casa Don Bosco (1996), Proyecto Educativo Pastoral, Valencia. Venezuela.

5. Dunn, William N. (1994), Public policy analysis. An Introduction. Englewood Cliff, 
NJ .: Prentice Hall. $2^{\text {nd }}$ edition.

6. FUNDA-ICI / FUNDACIÓN DE WAAL (1998), Niños de la Calle y Niños en Mendicidad en Venezuela. $N^{\circ}$ 2. Caracas. Venezuela.

7. Macrae, Duncan (1985), Policy indicators: Links between social science and public debate. Chapel Hill: University of North Carolina Press. USA.

8. UNICEF (1992), Análisis de Situación de Menores en Circunstancias Especialmente Difíciles. Nㅜ 6, Bogotá. Colombia.

9. Weiss, Carol H. (1998), Evaluation: Methods for studying programs and policies. Second Edition. Upper Saddle River, New Jersey: Prentice Hall. USA.

10. White, Louise (1994), Values, Ethics, and Standards in Policy Analysis. Encyclopedia of Policy Studies. $2^{\text {nd }}$ Edition. 